\title{
Ewigkeitsgarantien im europäischen Strafrecht - Ein Appell an die deutsche Volksvertretung ${ }^{1}$
}

\section{A. Vorbemerkung}

Ich sehe meine Aufgabe nicht darin, die gestellten Fragen durch eine Nacherzählung des Wortlauts der einschlägigen Normen der früheren oder projektierten Verträge oder des Inhalts dazu ergangener Entscheidungen des EuGH oder des BVerfG sowie der dazu bisher beobachteten Praxis der europäischen Organe zu beantworten. Denn ich sehe den Unterausschuss »Europarecht« des Rechtsausschusses des Deutschen Bundestages als den Repräsentanten des Souveräns der deutschen Demokratie, deren Parlament in Kürze in souveräner Freiheit über den für die Zukunft des Rechtsstaates in der Europäischen Union schicksalhaften Vertrag von Lissabon zu entscheiden haben wird. Die vornehmste Aufgabe der Strafrechtswissenschaft besteht deshalb im Augenblick darin, die spezifischen gesteigerten Legitimationsanforderungen, die in einem demokratischen Rechtsstaat an die Strafrechtsordnung gestellt werden müssen, in einer von Euphemismen und Halbherzigkeiten befreiten Deutlichkeit und ohne Verzagtheit vor politischen Machtstrukturen zu vermitteln sowie die bisherigen europäischen Akte und die durch den Vertrag von Lissabon ${ }^{2}$ geplante, speziell im Strafrecht tiefgreifende Neustrukturierung daran zu messen.

Ich werde deshalb zunächst in aller Kürze diejenigen Grundsätze zusammenfassen, die die Minimalia eines rechtsstaatlichen Strafrechts bilden und über die deshalb auch um noch so nützlicher europäischer Effekte willen nicht verfügt werden darf. Daraus werden sich die Antworten auf die einzelnen mir gestellten Fragen ebenso prägnant wie zwangsläufig ergeben.

\section{B. Mindestbedingungen der Strafrechtsordnung in einem demokratischen Rechtsstaat}

\section{Das Gesetzlichkeitsprinzip (nullum crimen, nulla poena sine lege parlamentaria)}

Dass die Angelegenheiten des Strafrechts durch den Gesetzgeber geregelt werden müssen, bei dem es sich in einer Demokratie nur um das Parlament handeln kann, ist ein allen geschriebenen Verfassungen voraus- und zugrundeliegendes Postulat, das bereits von den Staatsphilosophen und Strafrechtstheoretikern der Aufklärungszeit herausgearbeitet worden ist und inzwischen auch im Rechtskreis des Common Law vorbehaltlich gewisser Ausnahmen ${ }^{3}$ allgemeine Anerkennung genießt ${ }^{4}$. Dass dieser Grundsatz nur respek-

1 Stellungnahme zu den Fragen für das Expertengespräch im Unterausschuss Europarecht des Rechtsausschusses des Deutschen Bundestages am 28. 11. 2007 »Entsteht ein einheitliches europäisches Strafrecht?«.

2 13.12.2007, Amtsblatt der EU Nr. C 306 vom 17.12.2007.

3 Näher LK/Dannecker, § 1 Rdn. 45.

4 LK/Dannecker, § 1 Rdn. 5 ff., 43 ff.; Lüderssen, GA 2003, 71 ff. 
tiert wird, wenn das Parlament über die Strafgesetze in souveräner Freiheit befindet, ist eine analytische Aussage und deshalb unbestreitbar. Dass dies im Vertrag von Amsterdam $^{5}$ bei Einführung der Rahmenbeschlüsse ${ }^{6}$ in der sog. Dritten Säule missachtet und seitdem zumeist übersehen (oder sogar geflissentlich ignoriert) worden ist, ${ }^{7}$ ändert an der Wahrheit dieses Satzes so wenig, wie der Jahrtausende alte Irrglaube an das ptolemäische Weltbild den Kreislauf der Erde um die Sonne hinwegzueskamotieren vermochte.

\section{Das Strafrecht wird durch seine Aufgabe als ultima ratio zum Rechtsgüterschutz. legitimiert und limitiert}

Die Beschränkung des Strafrechts auf die Verhütung von Sozialschäden, die mit der in Deutschland üblichen Formel der ultima ratio zum Rechtsgüterschutz ${ }^{8}$ ausgedrückt wird (ähnlich, aber enger das angelsächsische harm principle ${ }^{9}$ ), stammt ebenfalls aus der Auf-

5 2.10.1997, Amtsblatt Nr. C 340 vom 10. November 1997.

6 Die gem. Art. 34 II, 2 b) EUV vom Rat erlassen werden, die Angleichung der Rechtsvorschriften der Mitgliedstaaten zum Gegenstand haben können und für diese hinsichtlich des zu erreichenden Ziels verbindlich sein sollen.

7 Vgl. allerdings bereits Albrecht/Braum/Frankenberg/Günther/Naucke/Simitis, KritV 2001, 279, 285 ff.; Deutscher, Die Kompetenzen der Europäischen Gemeinschaften zur originären Strafgesetzgebung, 2000, S. 371 ff.; w. N. b. Satzger, Die Europäisierung des Strafrechts, 2001, S. 451 Fn. 1936. Widersprüchlich Böse, GA 2006, 211, 216 ff., der das fundamentale demokratische Defizit einerseits erkennt, es aber andererseits mit dem Zirkelschluss überspielt, so sei nun einmal die EU konstruiert. Besonders eklatant wird dieser Selbstwiderspruch durch den beifälligen Hinweis auf den kryptischen Satz des BVerfG, die Mitgliedstaaten behielten über die Umsetzung der Rahmenbeschlüsse in innerstaatliches Recht die politische Gestaltungsmacht durch die Möglichkeit einer Verweigerung (S. 216), während einen Absatz später ausgeführt wird, die Entscheidungsfreiheit des nationalen Gesetzgebers sei normativ eben gerade nicht mehr gegeben (S. 217). Im Standardschrifttum wird die Problematik nicht behandelt, sondern nur die (für die demokratische Legitimation zweitrangige) fehlende unmittelbare Wirkung der Rahmenbeschlüsse hervorgehoben, s. Ambos, Internationales Strafrecht, 2006, S. 408 f.; Satzger Internationales und Europäisches Strafrecht, 2. Aufl 2008, S. 115; Hecker Europäisches Strafrecht 2. Aufl. 2007 § 5 Rn. 71; Streinz, Europarecht, 7. Aufl. 2005, S. 120; Calliess/Ruffert-Suhr, EUV/EGV Kommentar 3. Aufl. 2007, Art. 34 EUV Rn. 13; Braum, Europäische Strafgesetzlichkeit, 2003, S. 404 f. sieht mit Recht in den »Demokratiedefiziten im Rechtsetzungsverfahren ... eine unerträgliche Zumutung «, während Satzger, Die Europäisierung des Strafrechts, 2001, S. 451 ff. das Problem am Beispiel der Richtlinie (ähnlich S. 466 für Rahmenbeschlüsse) dadurch kompromisshaft zu lösen versucht, dass er für die Mitgliedstaaten »einen Umsetzungsspielraum von einigem Gewicht« verlangt, was aber das Defizit für den verbindlichen Teil nicht beseitigen kann. Zur Rspr. von BVerfG und EuGH s. u. C. 2.

8 Neuester Diskussionsstand in den Sammelbänden von Hefendehl/v. Hirsch/Wohlers (Hrsg.) Die Rechtsgutstheorie, 2003; v. Hirsch/Seelmann/Wohlers (Hrsg.), Mediating Principles, 2006, ibid. mein eigener Standpunkt S. 133 ff. bzw. S. 18 ff.; Roxin, in: Hefendehl (Hrsg.), Empirische und dogmatische Fundamente, kriminalpolitischer Impetus, 2005, S. 135 ff.; Hedendehl, GA 2007, $1 \mathrm{ff}$.

9 Zum Verhältnis näher v. Hirsch und Wittig, in: Die Rechtsgutstheorie (Fn. 7), S. 13 ff., 239 ff.; zur Ergänzung durch das Konzept des »Offensive Behaviour « v. Hirsch/Simester (Hrsg.), Incivilities, Oxford and Portland/Oregon 2006; grundlegend Feinberg, Harm to Others, New York 1984, und Offense to Others, ibid. 1985. 
klärungszeit und ist damit das Fundament der im 19. Jahrhundert siegreichen Rechtsstaatsbewegung gewesen, hinter die wir im 21. Jahrhundert nicht zurückfallen sollten.

III. Der Beschuldigte hat einen Anspruch auf ein faires Verfahren, und das setzt eine Ausstattung seiner Verteidigung mit effektiven Rechten zur Herstellung einer Balance zwischen Anklage und Verteidigung voraus

Das Recht des Beschuldigten auf einen sog. fair trial ist heute weltweit anerkannt ${ }^{10}$. Natürlich müssen diese Rechte auch der jeweiligen Verfahrensstruktur angepasst und dadurch insgesamt wirksam sein, so dass das im Europarecht wie eine Zauberformel benutzte französische Schlagwort des effet utile ${ }^{11}$ selbstverständlich auch für die faire Ausbalancierung des Strafverfahrens Geltung beansprucht.

\section{Bei diesen drei Pfeilern eines rechtsstaatlichen Strafrechts handelt es sich nicht um deutsche Spezialitäten, sondern um gemeineuropäische Traditionen}

Das skizzierte Dreipfeilermodell bedeutet wohlgemerkt keinen deutschen Sonderweg, sondern resultiert aus den besten gemeineuropäischen Traditionen seit der Aufklärung. Die Staatstheorie Montesquieus und die Strafrechtstheorie Beccarias haben an seiner Wiege gestanden, und der Grundsatz des fair trial ist ältestes angelsächsisches Gedankengut und hat etwa in der modernen italienischen Strafprozessordnung ${ }^{12}$ eine besonders markante Ausprägung erfahren. Die gelegentlich zu lesende Denunziation, eine Berufung darauf sei antieuropäisch und nationalistisch, beruht also auf einer totalen Verkennung gerade der wertvollsten europäischen Errungenschaften und geht in ihrer üblichen Adressierung an die deutsche Strafrechtswissenschaft schon deshalb fehl, weil gerade die Umgestaltung der deutschen Strafrechtspflege durch Gesetzgeber und Justiz seit geraumer Zeit ein besonders betrübliches Beispiel für den Abfall von den gemeineuropäischen Traditionen darstellt. Der Bundestag wird ja vermutlich in Kürze von der Bundesregierung mit einem Gesetzesentwurf zur Legalisierung der von der Justiz gegen das Gesetz etablierten Urteilsabsprachen im Strafverfahren konfrontiert und durch dessen schon heute absehbare Ignoranz gegenüber den Anforderungen einer fairen Verfahrensbalance überrascht werden, einer Ignoranz, die man auf zahlreichen internationalen Tagungen und Vortragsveranstaltungen der letzten Jahre geradezu als peinlich empfinden musste ${ }^{13}$. Wenn in der deutschen Strafrechtswissenschaft, aber beileibe nicht allein hier und übrigens auch längst nicht bei allen ihren Vertretern, in besonders intensiver Weise an der Bewahrung der besten europäischen Traditionen

10 Umfassend Weissbrodt/Wolfrum (Eds.), The Right to a Fair Trial, 1998; s. i. ü. Kühne, Strafprozessrecht, 7. Aufl. 2007, S. 174 ff.; Esser, Auf dem Weg zu einem europäischen Strafverfahrensrecht, 2002, S. 400 ff.; Trechsel, Human Rights in Criminal Proceedings, Oxford 2005, S. $83 \mathrm{ff}$.

11 Umfassend dazu Mosiek, Effet utile und Rechtsgemeinschaft, 2003.

12 Grdl. vor allem das strenge Verwertungsverbot bei rechtswidrig erlangten Beweisen in Art. 191 des Codice di procedura penale (v. 22. 9. 1988 m. zahlr. Änderungen).

13 Näher Schünemann, Wetterzeichen vom Untergang der deutschen Rechtskultur, 2005; ders., ZStW 119 (2007), 945, 950 ff. 
gearbeitet wird, möge also bitte niemand dem Missverständnis erliegen, darin artikuliere sich nationales Misstrauen gegenüber Europa; es ist genau umgekehrt.

\section{Beantwortung der gestellten Fragen}

»I. Woraus ergibt sich dem Grunde nach und in welchem Ausmaß die Kompetenz für die Schaffung eines einheitlichen europäischen Strafrechts: - nach dem Vertrag von Nizza, - nach der Rechtsprechung des EuGH, insbesondere in den Rechtssachen $C-176 / 03$ (»Umweltstrafrecht «) und C-440/05 (»Meeresverschmutzung«), - nach dem EU-Reformvertrag?«

1. Nach dem Vertrag von Nizza ergibt sich m. E. im Gegensatz zu einer verbreiteten Auffassung keine rechtswirksame Kompetenz der europäischen Organe zur Schaffung eines einheitlichen europäischen Strafrechts. Die Richtlinienkompetenz der EG ist zum einen sektoral beschränkt und sollte nach dem Willen der vertragschließenden Parteien die Strafgesetzgebung der Mitgliedstaaten unberührt lassen (Art. 280 IV, 2 EGV a fortiori). ${ }^{14}$ Die einzige nach ihrem Wortlaut für die Schaffung eines einheitlichen europäischen Strafrechts geeignete umfassende Kompetenzzuweisung, nämlich die seit dem Vertrag von Amsterdam in der sog. Dritten Säule vorgesehenen Rahmenbeschlüsse des Rates zur Angleichung der Rechts- und Verwaltungsvorschriften der Mitgliedstaaten gemäß Art. 34 II, 2 b) EUV, findet in dem für ein rechtsstaatlich-demokratisches Strafrecht fundamentalen Grundsatz nullum crimen, nulla poena sine lege parlamentaria ihre unübersteigbare Grenze. Denn wenn der Rat als Gremium von Regierungsmitgliedern, das bei der Fassung eines Rahmenbeschlusses ohne das europäische Parlament entscheidet, wirklich die nationalen Parlamente »hinsichtlich des zu erreichenden Ziels verbindlich« verpflichten könnten, wie es in Art. 34 EUV heißt, würde er deren souveräne Freiheit zerstören und sie zu »Lakaien von Brüssel« degradieren. Ich weiß, dass dies letztere von mir geprägte Wort ${ }^{15}$ von manchen als hässlich empfunden wird, aber hässlich ist in Wahrheit nicht das Wort, sondern die Realität, die damit bezeichnet wird: Das Strafrecht trennt den Bürger vom Verbrecher, den freien Menschen von der wie in einem Käfig gehaltenen Kreatur, und deshalb muss die Ausübung dieser furchtbarsten staatlichen Gewalt in einem Rechtsstaat allein dem demokratisch am direktesten und besten legitimierten parlamentarischen Gesetzgebungsprozess vorbehalten bleiben und darf nicht am Gängelband von Regierungsbeschlüssen geführt werden, die übrigens in der Praxis längst keine bloßen Rahmen mehr darstellen, zwischen dem den Parlamenten ein beträchtlicher Ausfüllungsspielraum bleibt, sondern eher Schießscharten mit einer winzigen Ausfüllungsritze für das Parla-

14 And. z. B. die eingehende Analyse von Satzger Europäisierung (Fn. 6), S. 405 ff., der aber gerade die »stärkste « Kompetenzzuweisung in Art. 280 EGV vor der Strafrechtsetzung enden lässt (S. 134, 138 ff.), was keinen Sinn machen würde, wenn eine Anweisungskompetenz qua Richtlinie den nationalen Gesetzgeber allgemein zu einem Ausführungsorgan degradieren und damit die Essenz der Gesetzgebung doch auf die Ebene der EG verlagern würde.

15 Schünemann, StV 2003, 531 ff.; ZRP 2003, 185 ff.; GA 2004, 193 ff. 
ment ${ }^{16}$. Die homöopathische Verdünnung parlamentarisch-demokratischer Machtausübungsstrukturen in den gubernativ-bürokratischen Institutionen der EG und EU mag politisch gesehen ihren nützlichen Effekt haben und ist ja auch in der Dogmatik des Europarechts mit Kategorien wie der »Mehrebenendemokratie « und der »gubernativen Rechtssetzung « behände aufgegriffen worden ${ }^{17}$. Im Strafrecht würde diese Abschilferung aber den Kern des demokratisch-gewaltenteilenden Rechtsstaats verletzen, deshalb haben die Väter der europäischen Verträge das Strafrecht aus den gubernativen europäischen Machtstrukturen zumindest intuitiv richtig bis 1996 herausgehalten, und dass die Sonderrolle des Strafrechts mit zunehmender Dominanz der Sicherheitsüber die Freiheitsperspektive zuerst bei den Politikern und dann offenbar auch in der Dogmatik des Europarechts in Vergessenheit geraten war, kann die Restauration vordemokratischen Gedankenguts zwar vielleicht erklären, aber nicht legitimieren.

2. Freilich scheint die Strafrechtswissenschaft erst wie die von Hegel apostrophierte Eule der Minerva in der Dämmerung ihren Flug begonnen zu haben, denn es gibt ja inzwischen eine ganze Serie von Rahmenbeschlüssen, die nicht zuletzt auch der Deutsche Bundestag treu und brav befolgt hat ${ }^{18}$, so dass es gegen jede Erfahrung in Politik und Geschichte wäre, wenn kritische Stimmen der Rechtswissenschaft jetzt noch Gehör finden würden. Aber überraschenderweise ist in dieser Hinsicht wegen eines eigenartigen doppelten Zickzackkurses sowohl des BVerfG als auch des EuGH noch gar nicht aller Tage Abend. Das soll aber nur ganz kurz skizziert werden, weil die gegenwärtige unübersichtliche Rechtslage womöglich durch den Vertrag von Lissabon bald obsolet sein wird.

a) Das BVerfG hat in seiner in vielerlei Hinsicht zwiespältigen Entscheidung zur Nichtigkeit des ersten EuHbG einerseits das Institut der Rahmenbeschlüsse gelobt und deren Bindungswirkung bejaht, andererseits aber ohne Auflösung des darin liegenden semantischen Widerspruchs ausgesprochen, das Parlament müsse in normativer Freiheit über die ihm in einem Rahmenbeschluss abverlangte Gesetzgebung entscheiden ${ }^{19}$. $\mathrm{Da}$ es ferner das erste EuHbG insgesamt kassiert und damit eigentlich eine über den eigenen Begründungsansatz hinausgehende Rechtsfolge angeordnet hat ${ }^{20}$, muss es als

16 Instruktive Übersicht bei Ambos (Fn. 6), S. 410 ff; Hecker (Fn. 6) § 11 Rn. 11 ff.

17 Zur »Mehrebenendemokratie« vgl. Brunkhorst/Kettner (Hrsg.), Globalisierung und Demokratie, 2000; Hiebaum, Politische Vergemeinschaftung unter Globalisierungsbedingungen, 1997; Zürn, PVS 37 (1996), 27 ff.; Jachtenfuchs/Kohler-Koch, in: dies. (Hrsg.), Europäische Integration, 1996, S. 15 ff.; Schuppert, in: Heyde/Schaber (Hrsg.), Demokratisches Regieren in Europa?, 2000, S. 65, 75 f.; Vogel, ZStW 116 (2004), 400 f. Programmatisch zur »gubernativen Rechtssetzung « die gleichnamige Monographie von v. Bogdandy (2000), in der das Strafrecht aber bezeichnenderweise überhaupt nicht vorkommt. Ohne Beachtung der demokratischen Voraussetzungen speziell der Strafgesetzgebung auch Tiedtke, Demokratie in der Europäischen Union, 2005.

18 Vgl. dazu oben Fn. 15.

19 Denn es hat in BVerfGE 113, 273, 301 betont, dass der Gesetzgeber die politische Gestaltungsmacht behalte und notfalls die Umsetzung verweigern könne, und spricht auf Seite 315 ausdrücklich davon, dass der deutsche Gesetzgeber »in normativer Freiheit unter Beachtung der verfassungsrechtlichen Maßstäbe« über die Ausübung des Gesetzesvorbehaltes in Art. 16 II 2 GG entscheiden können muss.

20 Insoweit zutr. Vogel, JZ 2005, 801, 804; Sondervotum Lübbe-Wolf, BVerfGE 113, 327 ff. 
offen angesehen werden, wie es über die Reichweite der Rahmenbeschlusskompetenz urteilen wird, wenn es einmal hart auf hart kommen sollte. Aber nachdem der Deutsche Bundestag beim zweiten EuHbG ${ }^{21}$ die ihm vom BVerfG eröffneten Spielräume, getreu den Vorgaben des Regierungsentwurfes, nur in dem geringst möglichen Umfange genutzt hat, scheint diese Frage gegenwärtig im Parlament nur auf geringes Interesse zu stoßen und soll deshalb heute von mir auch nicht vertieft werden. ${ }^{22}$

b) In gewisser Weise noch faszinierender ist die Rechtsprechung des EuGH, die die demokratischen Legitimationsprobleme der Rahmenbeschlüsse nicht erkannt hat, das Ganze als ein inneres Kompetenzproblem der EU aufgezäumt und dadurch unversehens in eine Art Echternacher Springprozession hineingeraten ist ${ }^{23}$. Wenig bekannt und auch in der Fragestellung nicht erwähnt ist zunächst die Entscheidung, die der EuGH auf Vorlage des Belgischen Schiedshofes zum Rahmenbeschluss über den europäischen Haftbefehl erlassen und in der er die fundierten rechtsstaatlichen Bedenken des Schiedshofes ohne viel Federlesen vom Tisch gewischt hat ${ }^{24}$. Diese Entscheidung wirkt auch deshalb überraschend, weil der EuGH kurz vorher in seiner Entscheidung zum Umweltstrafrecht auf allen Politikfeldern der EG eine strafrechtliche Annexkompetenz bejaht hatte, die durch Richtlinien wahrzunehmen wäre und eine Ausübung der dem Rat im dritten Pfeiler verliehenen Kompetenz zu Rahmenbeschlüssen blockieren soll $^{25}$. Allerdings soll diese Richtlinienkompetenz nach der neuen Entscheidung des EuGH zum Rahmenbeschluss »Meeresverschmutzung $\aleph^{26}$ nicht $»$ die Bestimmung von Art und Maß der anzuwendenden strafrechtlichen Sanktionen « umfassen (Tz. 70), so dass diesbezügliche Bestimmungen eines Rahmenbeschlusses nicht gegen Art. 47 EUV verstoßen würden (Tz. 71), im konkreten Fall jedoch wegen ihrer untrennbaren Verbindung mit den Bestimmungen über die Straftaten selbst ebenfalls nichtig seien (Tz. $72-74)$.

Es wird sich kaum bestreiten lassen, dass der EuGH durch die Erfindung einer strafrechtlichen Annexkompetenz der EG die Befugnisse der Judikative zur Interpretation und nicht zur Fortschreibung der europäischen Verträge deutlich überschritten haben dürfte ${ }^{27}$, ebenso wie durch die Eliminierung von Bestimmungen über Art und Maß der anzuwendenden strafrechtlichen Sanktionen aus der Richtlinienkompetenz der EG ohne grundsätzliche Bedenken gegenüber ihrer Domizilierung in der Rahmenbe-

21 Vom 20.07.2006, BGBl. I 1721.

22 Meinen Standpunkt zur Entscheidung des BVerfG und zu dessen Verarbeitung im zweiten EuHbG habe ich in StV 2005, 681 ff. sowie in: Joerden/Szwarc (Hrsg.), Europäisierung des Strafrechts in Polen und Deutschland - Rechtsstaatliche Grundlagen, 2007, S. 265 ff. näher dargelegt.

23 Womit ich die 1947 definitiv abgeschaffte Form meine, jeweils drei Schritte vor und zwei zurückzuspringen.

24 Verfassungsgerichtshof Belgien, Urteil Nr. 124/2005 vom 13.07.2005, zu finden unter www.arbitrage.be; EuGH Urteil vom 03.05.2007, NJW 2007, $2237 \mathrm{ff.}$

25 EuGH Urteil vom 13.09.2005, C-176/03, ABl. C. 315 vom 10.12.2005, S. 2; Entscheidung mit Gründen zu finden unter: http://curia.europa.eu - vgl. dort insbes. Tz. $531 \mathrm{ff}$.

26 EUGH Urteil vom 23.10.2007, C-440/05, EuGRZ 2007, 696 ff.

27 Vgl. zur Kritik nur Hefendehl, in: Joerden/Szwarc (Fn. 21), S. 41 ff.; Satzger Europäisches Strafrecht (Fn. 6), S. $111 \mathrm{ff}$. 
schlusskompetenz des Rates ein sonderbarer Kompetenz-Flickenteppich entstanden ist. Dass diese Lösung in irgendeiner Weise praktikabel ist, kann ich mir schwer vorstellen. Als geradezu niederschmetternd empfinde ich dabei die Leichtigkeit des Parlando-Stils, mit dem der EuGH das Strafrecht als eine Annexmaterie zu irgendwelchen Politiken qualifiziert und damit dessen qualifizierte Legitimationsanforderungen ignoriert. Die vom EuGH offenbar tolerierte Festsetzung von Art und Maß der Strafen durch einen Rahmenbeschluss von Regierungsorganen kann es in einem demokratischen Rechtsstaat nicht geben, und deswegen könnte der Deutsche Bundestag wegen der Ewigkeitsgarantie des Art. 79 Abs. 3 GG zur Durchführung eines solchen Beschlusses auch nicht verpflichtet sein.

3. Freilich braucht man sich bei diesen Fragen vielleicht nicht länger aufzuhalten, weil ja zukünftig in Art. 82, 83 AEUV eine grundsätzlich neue Regelung vorgesehen ist, die den Erlass von Mindestvorschriften zur Festlegung von Straftaten und Strafen durch von Europäischem Parlament und Rat nach dem ordentlichen Gesetzgebungsverfahren erlassene Richtlinien vorsieht. Es ist nicht zu bezweifeln, dass die demokratische Legitimation für die Vereinheitlichung des europäischen Strafrechts hierdurch gegenüber der geltenden Vertragslage wesentlich verbessert wird, weil nunmehr anders als bei den Rahmenbeschlüssen das Europäische Parlament entsprechend Art. 294 AEUV in den Gesetzgebungsprozess als gleichberechtigter Partner eingeschlossen ist. $\mathrm{Ob}$ das bereits genügt, um alle Legitimitätsbedenken zu zerstreuen, ist anhand der nachfolgenden Fragen zu prüfen.

\section{»II. Ergeben sich aus dem Grundgesetz Schranken, die die Geltung oder Anwendung eines einheitlichen europäischen Strafrechts begrenzen oder verhindern könn- ten?«}

1. Grundsätzlich kann ich hier auf die obige Skizze unter B. verweisen. Die dort beschriebenen drei Pfeiler eines rechtsstaatlichen Strafrechts nehmen an der Ewigkeitsgarantie von Art. 79 Abs. 3 GG teil, so dass eine Missachtung der sich daraus ergebenden Anforderungen in einer europäischen Richtlinie nicht in deutsches Recht transformiert werden dürfte. Weil inhaltliche Konflikte noch in den folgenden Fragen zu thematisieren sind, braucht an dieser Stelle nur die Grundsatzfrage beantwortet zu werden, ob die Festlegung von Mindestvorschriften, also der Zwang zur Kriminalisierung, wenigstens im Rahmen der künftigen europäischen Gesetzgebung ohne Verletzung des Demokratieprinzips ergehen könnte oder ob die Verbesserungen im Vertrag von Lissabon immer noch unzulänglich sind. Denn es ist zu bedenken, dass der Grundsatz der Wahlrechtsgleichheit für das Europäische Parlament weiterhin nicht vollständig verwirklicht sein wird, weil gem. Art. 14 Abs. 2 EUV-Lissabon die Zahl der Abgeordneten auf 750 und die eines einzelnen Mitgliedstaates auf 96 beschränkt ist, was sich eo ipso zum Nachteil des bevölkerungsstärksten Mitgliedstaates auswirkt. Es geht aber nicht nur um die formale Wahlrechtsgleichheit, sondern auch um ein in der politischen Wissenschaft unbestrittenes, sozusagen organisches Demokratiedefizit der Europäischen Union wegen der auf absehbare Zeit nicht zu behebenden Nichtexistenz einer einheitlichen europäischen Öffentlichkeit. Es ist ja gerade die entscheidende Anteilnahme der Öffentlichkeit am Gesetzgebungsprozess im Unterschied zu den 
prinzipiell geheim ablaufenden gubernativen Prozessen, die von den Apologeten der gubernativen Rechtssetzung vollständig übersehen wird, die aber als integraler Bestandteil einer wahrhaft demokratischen Gesetzgebung jedenfalls im Strafrecht unverzichtbar ist. Dass dieser Gesichtspunkt für eine an genuin demokratischen Werten orientierte und nicht durch Hurraeuropäismus getrübte Analyse von zentraler Bedeutung ist, scheint mir ebenso evident zu sein wie die Unmöglichkeit, ihn im vorliegenden Zusammenhang zu vertiefen. Zwar liegt die materielle demokratische Substanz von Entscheidungen des Europäischen Parlaments sicherlich signifikant über derjenigen von Rahmenbeschlüssen des Europäischen Rates, auf dessen mittelbarer, in den bürokratischen Entscheidungsstrukturen aber homöopathisch verdünnter demokratischer Legitimation deren Apologeten so gern herumreiten. Aber genau so liegt sie auch signifikant unter derjenigen eines nationalen Parlaments, das seine Beschlüsse vor der lebendigen demokratischen Öffentlichkeit rechtfertigen muss und deshalb auch nur in direkter Auseinandersetzung mit dieser zustande bringen kann.

2. Aus dem hieraus resultierenden Dilemma, dass selbst die verbesserte demokratische Legitimation nach dem Vertrag von Lissabon für das Strafrecht zu kurz greift, kann man allerdings einen Ausweg finden, wenn man die im Vertrag von Lissabon (Art. 82 Abs. 3 und 83 Abs. 3 AEUV) in ingeniöser Weise vorgesehene sog. »Notbremse « (emergency break) mit der ohnehin unabweisbaren Verstärkung der Rechte des Deutschen Bundestages gegenüber der Bundesregierung im europäischen Gesetzgebungsverfahren kombiniert (auf die Einzelheiten werde ich sogleich bei den anderen Fragen eingehen). Und damit wäre sichergestellt, dass der einzelne Bürger nur nach einer solchen Strafrechtsnorm in eine »wie in einem Käfig gehaltene Kreatur« verwandelt werden kann, an deren Verabschiedung mitzuwirken er eine dem Gleichheitsgrundsatz gehorchende Chance hatte - als Fundamentalpostulat eines demokratischen Strafrechts, aus dem etwa auch die Rechtsstaatswidrigkeit der Preisgabe des Prinzips der beiderseitigen Strafbarkeit im europäischen Haftbefehl folgt $t^{28}$.

\section{»III. Welche Instanz soll an Hand welchen Maßstabs die Rechtmäßigkeit der Normen eines einheitlichen europäischen Strafrechts überprüfen: - der nationale Gesetz- geber, - die nationalen Gerichte und das Bundesverfassungsgericht, - der EuGH, - der EGMR? «}

Natürlich sind alle vorgenannten europäischen oder staatlichen Organe nach Maßgabe und im Rahmen ihrer jeweiligen Kompetenz zur Überprüfung der Normen eines ein-

28 Wenn Weigend, in: FS Jung, 2007, 1069, 1073 meinen »demokratischen« Einwand gegen die Aufhebung des Erfordernisses der beiderseitigen Strafbarkeit im Auslieferungsrecht durch den $\mathrm{RbEuHb}$ und das EuHbG, nämlich dass der inländische Staatsbürger nicht zur Aburteilung wegen eines von ihm nicht beeinflussbaren ausländischen, in Deutschland kein Gegenstück aufweisenden Strafgesetzes ausgeliefert werden dürfe (StV 2005, 681, 683), wegen der »unbestrittenen Geltung des deutschen Strafrechts auch für Ausländer« für nicht näher erörterungsbedürftig erklären zu können verneint, so hat er dabei unversehens die Beurteilungsobjekte verwechselt. Denn es geht ja bei der Auslieferung um die Preisgabe des eigenen Staatsbürgers zur Bestrafung nach ausländischen Normen, deren inhaltliche Legitimität unter seiner demokratischen Mitwirkung im Inland gerade verneint wurde. 
heitlichen europäischen Strafrechts berufen, wobei (solange die unmittelbar anwendbaren Normen ja weiterhin dem nationalen Recht angehören) dies von den nationalen Gerichten nur durch Vorlagebeschlüsse ans BVerfG oder an den EuGH geschehen könnte. Solange es sich nach wie vor um nationale Rechtsakte handelt, wird der EGMR im bisherigen Rahmen zuständig bleiben, der für richtungsweisende Entscheidungen geeignet ist, als alltäglicher Rechtsschutz aber aus zahlreichen Gründen überfordert wäre. Über die Möglichkeiten zu einer Kontrolle durch den EuGH will ich mich hier nicht weiter verbreiten, weil ich auf absehbare Zeit in eine Exekutierung der oben unter B. angeführten drei Pfeiler durch den EuGH keine realistischen Erwartungen setze. Wer Strafrecht ohne Bedenken als Annexkompetenz beliebiger europäischer Politiken ansieht und in puncto demokratischer Legitimation von vornherein keine Bedenken hat, wird vermutlich auch für deren Einzelheiten nicht das notwendige Verständnis aufbringen. Wenigstens an dieser Stelle darf ich deshalb im Interesse der wissenschaftlichen Ehrlichkeit nicht unterschlagen, dass ich dem EuGH die ausreichende Legitimation für eine Schleifung der drei über Jahrhunderte aufgerichteten Pfeiler des europäischen Rechtsstaates ausdrücklich bestreiten möchte ${ }^{29}$. So ruhen also die Hoffnungen, wenn man einmal von dem angesichts seines janusköpfigen Urteils zum europäischen Haftbefehl für die Zukunft schwer auszurechnenden BVerfG absieht, auf dem Deutschen Bundestag als Verteidiger eines rechtsstaatlich demokratischen Strafrechts in Europa. Ich hatte bereits bei dem 2004 gemeinsam mit 10 Kollegen publizierten »Alternativentwurf Europäische Strafverfolgung « vorgeschlagen, zur Behebung des demokratischen Defizits der Rahmenbeschlüsse die Bundesregierung an die Stellungnahme des Bundestages zu binden ${ }^{30}$, so wie dies auch in etlichen nordund nordwesteuropäischen Mitgliedstaaten der Fall ist ${ }^{31}$ und durch II, 4 der Vereinbarung zwischen Bundestag und Bundesregierung in Ausführung von $\S 6 \mathrm{EUZBBG}^{32}$ als »Parlamentsvorbehalt« im Prinzip vorgesehen ist ${ }^{33}$, jedoch dann versagt, wenn die Bundesregierung »aus wichtigen außen- oder integrationspolitischen Gründen« eine abweichende Entscheidung trifft. Es sollte deshalb jetzt eine entsprechende Vorschrift, jedoch ohne eine Prärogative der Bundesregierung, für die Ausübung der »Notbremse« gemäß Art. 82 Abs. 3 und 83 Abs. 3 AEUV in das Grundgesetz aufgenommen werden, um dem Bundestag eine Vorab-Kontrollkompetenz über die Rechtsstaatlichkeit der Normen eines einheitlichen europäischen Strafrechts zu verschaffen und damit auch jene demokratische Legitimation im Wege einer prästabilierten Harmonie zu schaffen, die sonst den europäischen Rechtsakten im Strafrecht abgehen würde.

$29 \mathrm{Zu}$ den Legitimationsproblemen des EuGH selbst siehe Mähner, Der Europäische Gerichtshof als Gericht, 2005; Balders/Hansack, ZRP 2006, 54.

30 Schünemann (Hrsg.), Alternativentwurf Europäische Strafverfolgung, 2004, S. 24 f.

31 Rohlff, Der Europäische Haftbefehl, 2003, S. 23.

32 EUZBBGVbg, Fassung vom 28. 9. 2006, BGB1 I S. 2177.

$33 \mathrm{Vgl}$. auch den von den Unionsfraktionen noch in ihrer Zeit als Opposition in den Bundestag eingebrachten Gesetzesentwurf BT-Drs. 15/4716, der der Diskontinuität zum Opfer gefallen und seitdem nicht erneuert worden ist. 
Der Deutsche Bundestag hat auch die Macht, diese Ergänzung des Grundgesetzes vor der Ratifikation des Lissabon-Vertrages durchzusetzen. Wenn er diese Chance verabsäumt, wird er sich nicht nur an allen Errungenschaften der strafrechtlichen Aufklärung in Europa seit Montesquieu, Beccaria, Hommel und Feuerbach versündigen, sondern auch eine Selbstkastration auf dem empfindlichsten Feld des demokratischen Rechtsstaates vornehmen, wie sie in anderer Form nur vom Kirchenvater Origenes überliefert ist.

\section{IV. »Vor welchem Gericht und auf welcher Grundlage können Beschuldigte Rechts- schutz erlangen? «}

1. Diese Frage zielt auf den Grundsatz der gegenseitigen Anerkennung, der nunmehr in Art. 82 Abs. 1 AEUV endgültig als Fundament der Strafjustiz in Europa festgeschrieben werden soll. Ich habe mich hierzu oft genug kritisch geäußert und will nur bemerken, dass dieser Grundsatz in Verbindung mit der ebenfalls festzuschreibenden Kompetenz zur Statuierung von Mindestvorschriften zur Festlegung von Straftaten und Strafen gem. Art. 83 Abs. 1 AEUV eine Tendenz zur permanenten Verschärfung des Strafrechts und zur europaweiten Exekutierbarkeit des jeweils punitivsten Strafrechts normativ verankert und auf die Dauer den verheißenen Raum der Freiheit, der Sicherheit und des Rechts in, wie Schiller es durch den Marquis Posa formuliert hat, die Ruhe eines Kirchhofs zu verwandeln droht. ${ }^{34}$

2. Immerhin sieht Art. 82 Abs. II 2 b AEUV weiterhin Richtlinien zu Mindestvorschriften über die Rechte des einzelnen im Strafverfahren vor, deren Ausarbeitung nach verheißungsvollem Beginn leider bis heute kläglich gescheitert ist ${ }^{35}$. Auch hier bietet also der Vertrag von Lissabon einen Silberstreif am Horizont, doch möchte ich den Hinweis nicht unterschlagen, dass mit der Garantie einzelner Rechte ein insgesamt faires und d. h. ausbalanciertes Strafverfahren noch nicht erreicht werden kann. Dieses Ziel versucht der von mir gemeinsam mit 15 europäischen Kollegen ausgearbeitete »Alternativentwurf Europa II « modellhaft abzubilden, weshalb ich mich im Einzelnen darauf beziehen möchte. ${ }^{36}$

\section{$» V$. Welche Auswirkungen auf das nationale Strafrecht sind von einem einheitlichen europäischen Strafrecht zu erwarten?"}

Wie bereits meine Antwort zur vierten Frage deutlich macht, droht nach den bisherigen Konzepten eine Tendenz zur permanenten Ausdehnung des Strafrechts zu dominieren, die letzten Endes die von mir eingangs skizzierten drei Pfeiler niederreißen würde. Es gibt in meinen Augen nichts Verfehlteres als die Vorstellung des Strafrechts als einer Annexmaterie zu beliebigen Politiken und als das Verständnis von der Aufgabe Europas als eines permanenten Einpeitschers des nationalen Strafrechts. Nicht

34 Don Carlos, 3. Akt, 10. Auftritt.

35 Näher Schünemann, StV 2006, 361 ff.; offenbar endgültig gescheitert in der Justiz- und Innenministerkonferenz am 13. 6. 2007.

36 Schünemann (Hrsg.), Ein Gesamtkonzept für die europäische Strafrechtspflege, 2006. 
weniger falsch ist die häufig zu lesende Vorstellung, das Strafrecht sei wie kein anderes Rechtsgebiet von nationalen Eigentümlichkeiten und Traditionen gekennzeichnet, quasi nach Art des Schottenrocks und Dudelsacks oder des Stierkampfes in Spanien. Genau umgekehrt sind es ja die vornehmsten Traditionen seit der Zeit der strafrechtlichen Aufklärung, dass es im Strafrecht um die ultima ratio zum Rechtsgüterschutz (in anderer Formulierung: um die Verhütung von Sozialschäden) geht und nicht um die Bewahrung schrulliger nationaler Traditionen. Natürlich kann es eine nationale Verwurzelung spezifischer Rechtsgüter geben wie etwa die extrem begrenzten Wasserressourcen in semiariden Mitgliedstaaten, die in von Dauerregen heimgesuchten Ländern kein Pendant haben. Aber das ist die Ausnahme, denn in aller Regel kann es in einem einheitlichen Industrieraum mit einer im Großen und Ganzen auf dem gleichen Entwicklungsniveau angekommenen Kultur auch nur dieselben Rechtsgüter geben. Und es ist deshalb schon ein katastrophales Defizit, dass in der Grundrechtecharta der EU, die ja durch Art. 6 EUV gem. Lissabon-Vertrag verbindlich gemacht werden soll, zwar in Art. 49 Abs. 3 die Verhältnismäßigkeit der Strafen garantiert wird, auch in Art. 48 Abs. 1 die Unschuldsvermutung, dass aber eine Grundsatznorm über die Begrenzung der staatlichen Strafgewalt, die ja in Wahrheit von beiden Garantien logisch vorausgesetzt wird, vollständig fehlt. Wenn man bedenkt, wie vielen sekundären Freiheiten und Interessen in der Grundrechtecharta und den Politiken der EU eine ausgeprägte Aufmerksamkeit gezollt wird, so mochte die sonderbare Abstinenz im Bereich des Strafrechts allenfalls so lange hingehen, wie sich Europa auf diesem Gebiet keine eigene Kompetenz zuschrieb; aber seitdem das grundlegend anders geworden ist, hat die Abstinenz etwas Anstößiges bekommen. Auch wenn dieses Desiderat realistischerweise im Vertrag von Lissabon nicht mehr zu erfüllen sein wird, muss es doch jedenfalls in einem wahrhaften Raum der Freiheit und des Rechts die eiligste Folgemaßnahme bilden. Um so wichtiger wird deshalb die Rolle der nationalen Parlamente sein, sich bei der Ratifizierung des Lissabon-Vertrages die souveräne Entscheidung über die erwähnte »Notbremse« zu sichern und damit zukünftig jene Verteidigung der demokratischen und rechtsstaatlichen Grundlagen des Strafrechts sicherzustellen, die nach der bisherigen Erfahrung vom europäischen Gerichtshof und von den Regierungen nicht ohne weiteres zu erwarten sein wird.

\section{»VI. Welcher Handlungsbedarf ergibt sich für den nationalen Gesetzgeber im Zusam- menhang mit der Entstehung eines einheitlichen europäischen Strafrechts? «}

Die wirklich entscheidende Antwort findet sich bereits oben, denn allein die Sicherung der souveränen Entscheidung über die »Notbremse« wird den Parlamenten der Mitgliedstaaten auch auf der europäischen Ebene jene für die bürgerliche Freiheit entscheidende Aufgabe erhalten können, die sie seit der Durchsetzung der Demokratie gespielt haben und für deren Wahrnehmung die Organe der Europäischen Union, wie ich im einzelnen dargelegt habe, nicht hinreichend konzipiert oder prädestiniert sind. Und selbst die »weichen « Einflussmöglichkeiten, die den mitgliedstaatlichen Parlamenten auf den Gesetzgebungsprozess der EU im Vertrag von Lissabon eingeräumt werden, besitzen nur dann ein hinreichendes Gewicht, wenn die Parlamente auch souverän über den Ausnahmezustand, und d.h. konkret: über die »Notbremse« entscheiden können. 